\title{
Light scalar tetraquarks from a holographic perspective
}

\author{
Hilmar Forkel $^{1}$ \\ ${ }^{1}$ Institut für Physik, Humboldt-Universität zu Berlin, D-12489 Berlin, Germany
}

\begin{abstract}
We discuss how a dominant tetraquark component of the lightest scalar mesons may emerge in AdS/QCD gravity duals. In particular, we show that the exceptionally strong binding required to render the tetraquark ground state lighter than the lowest-lying scalar quark-antiquark nonet can be holographically encoded into bulk-mass corrections for the tetraquark's dual mode. The latter are argued to originate from the anomalous dimension of the corresponding four-quark interpolator. To provide a concrete example, we implement this mechanism into the dilaton soft-wall dual for holographic QCD. Preventing the lowest-lying dual mode from collapsing into the AdS boundary then establishes a rather generic lower bound on the tetraquark mass (which may be overcome in the presence of additional background fields). We further demonstrate that the higher tetraquark excitations can become heavier than their quark-antiquark counterparts and are thus likely to dissolve into the multiparticle continuum.
\end{abstract}

PACS numbers: 11.25.Tq, 11.25.Wx, 14.40.Cs, 12.40.Yx 


\section{INTRODUCTION}

The nature of the light scalar mesons remains a key challenge for our understanding of how QCD generates bound states. Exceptionally strong and diverse mixing patterns between the differently flavored quarkonium components and with scalar glueballs are among the intricacies which kept this sector exciting and controversial for more than four decades. Theoretical approaches to the subject include a long tradition of model building, methods based on dispersion relations and effective field theories, QCD sum-rule analyses and more recently devoted and unquenched lattice simulations. The corresponding literature may be traced at least partly from the reviews [1 5 . 5 .

In recent years, evidence has accumulated in favor of Jaffe's classic suggestion [6] that the lightest scalar meson nonet with masses below $1 \mathrm{GeV}$ (and potentially a first recurrence beyond $1 \mathrm{GeV}$ [7]) may contain a dominant (cryptoexotic) tetraquark component [4, 5, 811]. This at present arguably most convincing interpretation demands an explanation for the exceptionally strong binding between the four "valence" quarks, however, which is able to reduce the lightest tetraquark mass significantly below that of the scalar quark-antiquark ground state. With the advent of holographic strong-coupling techniques based on the gauge/gravity correspondence [12] and the construction of a first generation of approximate holographic QCD (or AdS/QCD) duals [13] it seems pertinent to explore whether those can provide new insights into existence and structure of strongly bound tetraquarks. In the present note we will take a first step in this direction.

Several holographic studies of the light spin-0 meson [14 16] (and glueball [17]) sectors in the AdS/QCD framework have recently appeared. Those were based exclusively on quark-antiquark interpolators, however, which presuppose a $\bar{q} q$-dominated meson structure. Moreover, the first two of them exclude the lightest scalars $\left(f_{0}(600)\right.$ and $\left.K_{0}^{*}(800)\right)$ from their analysis. In any case, it is rather obvious (and will be discussed in more detail below) that a straightforward extension of this approach to $\bar{q}^{2} q^{2}$ interpolators would describe four-quark states which are heavier, not lighter than the $\bar{q} q$ ground state. Our main focus will therefore be to search for a minimal but systematic AdS/QCD extension in which tetraquarks emerge naturally as the lightest scalar mesons. The sought-after holographic mechanism should further be able to render higher tetraquark excitations heavy (and thus broad) enough to avoid generating more low-lying scalar nonets than experimentally seen. 
In looking for a holographic representation of the required binding, we are guided by the expectation that the lightest scalar tetraquarks essentially consist of a "good" (i.e. maximally attractive) diquark and a good antidiquark [38] which form an $s$-wave bound state [6, 8, 10]. The exceptional lightness of the tetraquark ground state is then mainly a consequence of the good diquark's large (and potentially instanton-induced [19 21]) binding energy [22]. This suggests to adapt the approach introduced in Ref. [23], which describes systematic mass reductions among the light baryons due to good-diquark binding holographically in terms of bulk-mass corrections for their dual modes, to the problem. Below we will explore to what extent such corrections, as induced by the anomalous dimension of the corresponding four-quark interpolator, are able to generate light scalar tetraquarks as well.

\section{SCALAR SOFT-WALL DYNAMICS}

As a specific dynamical basis for our study we adopt the dilaton-soft-wall gravity dual of Ref. [24] which is governed by the action

$$
S^{(\mathrm{sw})}=\frac{1}{\kappa^{2}} \int d^{5} x \sqrt{|g|} e^{-\Phi} \operatorname{tr}\left\{|D X|^{2}-m_{5}^{2}|X|^{2}+\frac{1}{4 g_{5}^{2}}\left(F_{L}^{2}+F_{R}^{2}\right)\right\} .
$$

The gauge-covariant derivative $D_{M}=\partial_{M}-i A_{L, M}+i A_{R, M}$ contains the left/right flavor gauge potentials $A_{L / R, M}$ with field strengths $\left(F_{L / R}\right)_{M N}=\partial_{M} A_{L / R, N}-\partial_{N} A_{L / R, M}-$ $i\left[A_{L / R, M}, A_{L / R, N}\right]$. The spin-0 bulk field

$$
X=\left(X_{0}+\varphi^{0} t^{0}+\varphi^{a} t^{a}\right) e^{2 i \pi^{a} t^{a}}
$$

consists of the background field $X_{0}$ dual to the quark condensate, a flavor nonet $\varphi^{A}$ (with $A \in\{0, \ldots 8\}$ ) of scalar fluctuations (where the flavor singlet $\varphi^{0}$ may contain a glueball component) and the chiral pseudo-Goldstone bosons $\pi$. The $\mathrm{U}(3)$ flavor generators are normalized as $\operatorname{tr}\left\{t^{A} t^{B}\right\}=\delta^{A B} / 2$. The remaining, non-dynamical background fields comprise the $\mathrm{AdS}_{5}$ geometry

$$
d s^{2}=g_{M N} d x^{M} d x^{N}=\frac{R^{2}}{z^{2}}\left(\eta_{\mu \nu} d x^{\mu} d x^{\nu}-d z^{2}\right)
$$

with curvature radius $R$ (where $\eta$ is the four-dimensional Minkowski metric and $z$ the Poincaré coordinate of the fifth dimension) and the dilaton field

$$
\Phi(z)=\lambda^{2} z^{2}
$$


which is solely responsible for conformal-symmetry breaking in the infrared. The mass spectrum of the scalar meson nonets is then determined by the $\varphi$-dependent, bilinear part

$$
S[\varphi]=\frac{1}{2 \kappa^{2}} \int d^{5} x \sqrt{|g|} e^{-\Phi}\left[g^{M N} \partial_{M} \varphi \partial_{N} \varphi-m_{5}^{2} \varphi^{2}\right]
$$

of the bulk action (1). Since the dynamics (5) is flavor diagonal, we have dropped the index of the scalar fields. (The "inverted" flavor-breaking pattern in tetraquark nonets [10] is thus beyond the scope of the present study.)

Variation of the action (5) generates the field equation

$$
\left[\nabla_{M} \nabla^{M}-\left(\partial_{M} \Phi\right) \partial^{N}+m_{5}^{2}\right] \varphi=0
$$

where $\nabla_{M} \varphi=\left(\partial_{M}+\Gamma_{M N}^{N}\right) \varphi$ and $\Gamma_{M N}^{N}=\partial_{M} \ln |g|^{1 / 2}$. In terms of the four-dimensional Fourier transform

$$
\varphi(x, z)=\int \frac{d^{4} q}{(2 \pi)^{4}} e^{-i q x} \hat{\varphi}(q, z)
$$

the ensuing radial equation becomes

$$
\left[\partial_{z}^{2}-\left(\frac{3}{z}+2 \lambda^{2} z\right) \partial_{z}+q^{2}-\frac{R^{2} m_{5}^{2}}{z^{2}}\right] \hat{\varphi}(q, z)=0 .
$$

Redefining the scalar field as

$$
\hat{\varphi}(q, z)=\left(\frac{z}{R}\right)^{\frac{3}{2}} e^{\lambda^{2} z^{2} / 2} \phi(q, z)
$$

then eliminates the first-derivative term and turns Eq. (8) into the Sturm-Liouville problem

$$
\left[-\partial_{z}^{2}+V(z)\right] \phi(q, z)=q^{2} \phi(q, z)
$$

with the potential

$$
V(z)=\left(\frac{15}{4}+m_{5}^{2} R^{2}\right) \frac{1}{z^{2}}+\lambda^{2}\left(\lambda^{2} z^{2}+2\right) .
$$

This formulation is convenient for evaluating the discrete meson mass spectrum $m_{n}^{2}=q_{n}^{2}$ associated with the normalizable scalar bulk modes $\phi\left(m_{n}, z\right)$.

At this point, a few comments are in order on the application of current AdS/QCD duals to three-color (i.e. $N_{c}=3$ ) physics in general and to exotics in particular. The semiclassical treatment of the underlying string (or, at weak curvature, gravity) dynamics is justified for large $N_{c}$. Since calculating even the leading $1 / N_{c}$ corrections is beyond present capabilities, the extrapolation to $N_{c}=3$ has to remain naive and speculative. This holds in particular 
for bottom-up approaches where the $N_{c}$ dependence enters by comparison of calculated amplitudes with gauge-theory results [25], for example in bulk coupling and mass parameters (one finds e.g. $g_{5}^{2}=12 \pi^{2} / N_{c}$ for the gauge coupling in Eq. (11)), in the normalization of correlators, or when encoding the Yang-Mills $\beta$ function. From a formal perspective, the resulting extrapolation to smaller $N_{c}$ should therefore be regarded as a working hypothesis. In any case, it provides a consistent $N_{c}$ counting for the so far calculated observables and a semi-quantitative description of classical hadron properties (at the 10-30\% level).

One should keep in mind, however, that this naive treatment faces more challenging tasks in the exotic multiquark sector where the $N_{c}$ dependence can be particularly strong and where the extrapolation to $N_{c}=3$ becomes a practical necessity. Indeed, exotics are dissolving in the $N_{c} \rightarrow \infty$ limit [26] (tetraquarks perhaps already for $N_{c}>30-50$, as

unitarized chiral perturbation theory suggests [27]) and the higher-lying tetraquarks are expected to remain highly unstable under strong decay (with widths comparable to their masses) even at $N_{c}=3$ [6]. Our main focus will be on the lightest scalar tetraquark nonet, however, which should be relatively narrow (due to the paucity of open decay channels, limited phase space and partial dynamical suppression) and hopefully reasonably amenable to the naive $N_{c} \rightarrow 3$ extrapolation.

\section{INTERPOLATOR AND ANOMALOUS DIMENSION}

The AdS/CFT dictionary [12] relates the normalizable bulk modes $\phi$ to those interpolating fields of the dual gauge theory which have enhanced overlap with the corresponding hadrons. More specifically, in the scalar sector the conformal dimension $\Delta$ of a meson interpolator prescribes the mass term in the potential (11) as

$$
m_{5}^{2} R^{2}=\Delta(\Delta-4)
$$

and thereby sets the $z \rightarrow 0$ boundary condition for its dual mode, i.e. for the solution of Eq. (10). In the case of ordinary scalar mesons the quark-antiquark interpolator

$$
J_{\bar{q} q}^{A}=\bar{q}^{a} t^{A} q^{a}
$$

is (up to factors) the unique choice with $\Delta_{\bar{q} q}=3$. For light scalar mesons with a dominant tetraquark component, on the other hand, the larger number of possible couplings between 
the four quarks [28] allows for several potential tetraquark interpolators with the minimal classical scaling dimension $\Delta_{\bar{q}^{2} q^{2}}=6$. In bottom-up duals it is therefore not a priori clear how to assign states to these interpolators. In the light of our above comments a natural choice, containing a good diquark and a good antidiquark, would be

$$
J_{\bar{q}^{2} q^{2}}^{A}=\varepsilon^{a b c} \varepsilon^{a d e} \bar{q}^{b} C \Gamma^{A} \bar{q}^{c} q^{d} C \Gamma^{A} q^{e}
$$

where $C$ is the charge conjugation matrix, no sum over $A$ is implied and the spin-flavor matrices $\Gamma$ for the different members of the nonet may be found e.g. in Refs. [9, 20]. The explicit form (14) is just an illustrative example, however, and will not affect our results. Indeed, for our purpose it suffices to specify the tetraquark interpolator's quantum numbers, its scaling dimension and the defining property of maximal overlap with the tetraquark ground state.

The soft-wall meson spectrum resulting from the $\bar{q} q$ interpolator (13) is [14]

$$
m_{\bar{q} q, n}^{2}=4\left(n+\frac{3}{2}\right) \lambda^{2}
$$

for $n \geq 0$. The eigenmodes are $\phi_{\bar{q} q}\left(m_{n}, z\right) \propto(\lambda z)^{3}(z / R)^{-3 / 2} e^{-\lambda^{2} z^{2} / 2} L_{n}^{(1)}\left(\lambda^{2} z^{2}\right)$ where $L_{n}^{(\alpha)}$ is a generalized Laguerre polynomial [29]. The naive tetraquark spectrum corresponding to an interpolator like Eq. (14) with classical scaling dimension $\Delta_{\bar{q}^{2} q^{2}}=6$ (and no anomalous dimension), on the other hand, is

$$
m_{\Delta=6, n}^{2}=4(n+3) \lambda^{2} .
$$

The radial excitations are thus likewise organized into a linear square-mass trajectory with the universal slope $4 \lambda^{2}$. The corresponding normalizable eigenmode solutions are now

$$
\phi_{\Delta=6}\left(m_{n}, z\right)=N_{\Delta=6, n}(\lambda z)^{6}\left(\frac{z}{R}\right)^{-3 / 2} e^{-\lambda^{2} z^{2} / 2} L_{n}^{(4)}\left(\lambda^{2} z^{2}\right) .
$$

The intercept of the $\Delta=6$ trajectory is twice as large as that of its $\Delta=3$ counterpart, however, which implies $m_{\Delta=6,0}^{2} / m_{q \bar{q}, 0}^{2}=2$ for the ground states. This exemplifies the general case: in the absence of bulk interactions and when ignoring anomalous dimensions (which is so far common practice in AdS/QCD applications), the holographic meson-mass predictions increase with the interpolator dimension $\Delta\left(\right.$ as $m_{n}^{2}=4(n+\Delta / 2) \lambda^{2}$ in the dilaton soft-wall for $d=4)$. 
The above pattern adequately reproduces the empirical mass hierarchy among the less strongly bound $\bar{q} q$ mesons (with $\Delta=3$ ) and scalar glueballs (with $\Delta=4$ ). The exceptionally strong attraction required to reduce the masses of the lightest tetraquark nonet below those of the scalar $\bar{q} q$ ground state, on the other hand, is obviously not reflected in this minimal description. In the following we will examine whether the neglect of the tetraquark interpolator's anomalous dimension may be responsible for this shortcoming. As already mentioned, this would not be unexpected if the additional binding originates from the maximally attractive diquark channel because diquark-content-dependent anomalous dimensions of the nucleon interpolators were found to describe good-diquark binding effects in the light baryon spectrum [23] (on the basis of the "metric soft-wall" gravity dual of Ref. [30]). Indeed, the anomalous dimension of the tetraquark interpolator will generally depend on its diquark content as well, and thus on the diquark content of the scalar meson with which it has enhanced overlap. Moreover, if two quarks form a good diquark whenever possible, and if good diquarks systematically reduce the masses of the hadrons which they dominate (compared to those containing other types of diquarks), one would expect this behavior to have a universal representation in the gravity dual.

Holographically, the inclusion of the anomalous dimension $\gamma$ turns the mass term (12) for the bulk modes dual to the $\Delta_{\bar{q}^{2} q^{2}}=6$ interpolator into

$$
m_{5}^{2}(z) R^{2}=[6+\gamma(z)][2+\gamma(z)] .
$$

(In more complex gravity duals, mass corrections may further arise from couplings to additional, e.g. condensate-related bulk fields.) The $z$ dependence of $\gamma$ is inherited from its renormalization group (RG) flow $\gamma(\mu)$ since the AdS/CFT dictionary translates the RG scale $\mu$ into the inverse of the fifth dimension, i.e. $\mu \sim 1 / z$ [39]. Ideally, $\gamma(z)$ should be obtained from (both perturbative and nonperturbative) QCD and, as any other information from the gauge-theory side, implemented into the gravity dual in bottom-up fashion. However, at present this approach is ruled out by the absence of QCD information on $\gamma(z)$. As a preliminary substitute, we will therefore resort to a reasonable ansatz for $\gamma(z)$ and restrict it as tightly as possible by consistency, stability and physics requirements. This strategy cannot directly relate the ensuing mass corrections (18) to the anomalous dimension in $\mathrm{QCD}$, but it will provide quantitative orientation and general insights, especially on how much tetraquark binding the anomalous-dimension mechanism can generate in principle, and even lead to a 
rather unique semi-quantative guess for $\gamma$ which is essentially independent of the specific AdS/QCD dynamics (1) adopted to derive it. Nevertheless, this estimate will have to be checked against - and in case of significant disagreements replaced by - QCD results for $\gamma$ (e.g. from the lattice) when they eventually become available.

A glance at the soft-wall potential (11) with the generalized mass term (18) for $\Delta_{\bar{q}^{2} q^{2}}=$ 6 reveals that even a scale-independent anomalous dimension $\gamma<-3$ would reduce its repulsion sufficiently to bring the tetraquark ground-state mass down below that of the $\bar{q} q$ ground state. However, it would do the same for all radial excitations as well, whereas phenomenologically higher-lying tetraquark excitations (with the exception of the first one if interpreted as a recurrence of the tetraquark ground state [7]) should be pushed up toward or beyond the corresponding $\bar{q} q$ excitations, to be broad enough to explain the absence of additional scalar nonets in the measured meson spectrum. This suggests to check whether a suitably running $\gamma(z)$ can accommodate the phenomenological situation. As it turns out, the necessary qualitative requirements may be cast into the simple power-law behavior

$$
\gamma(z)=-a z^{\eta}+b z^{\kappa}
$$

with $\kappa \gg \eta$, so that the first (second) term modifies mainly the small- $z$ (large- $z$ ) region of the Sturm-Liouville potential. Moreover, the coefficients and exponents in Eq. (19) can be almost quantitatively determined by the underlying physics. To show this, we split the soft-wall potential (11) with the generalized mass term (18) as

$$
V_{q^{2} \bar{q}^{2}}(z)=\left(\frac{15}{4}+12\right) \frac{1}{z^{2}}+\lambda^{2}\left(\lambda^{2} z^{2}+2\right)+\Delta V(z)
$$

into the uncorrected (i.e. $\gamma=0$ ) potential with the spectrum (16) and the finite- $\gamma$ correction

$$
\Delta V(z)=\gamma(z)[\gamma(z)+8] \frac{1}{z^{2}}
$$

Maintaining asymptotically linear trajectories and enough repulsion to move the masses of the higher excitations upward restricts the exponent $\kappa$ to $\kappa \lesssim 2$. Approximate slope universality [31] further demands $|b| \ll 1$. The collapse of solutions to the mode equation (10) into the $\mathrm{AdS}_{5}$ boundary at $z \rightarrow 0$ [32] is avoided for $\eta>0$ (see below), and maximal attraction at smaller $z$, where the $z^{-2}$ enhancement reduces the ground-state mass most effectively, demands $\eta \ll 1$. As a consequence, Eq. (19) does not affect the AdS/CFT boundary condition of the tetraquark bulk modes for $z \rightarrow 0$ since $\gamma(0)=0$, in accord with 
asymptotic freedom. Maximizing the overall attraction of the potential correction (21), finally, requires $a \simeq 4$ (see below). It is worth noting that anomalous dimensions of a $z$ dependence qualitatively similar to Eq. (19) (in the region of interest) have recently been found in dual backgrounds which are of holographic RG-flow type [33]. (For a holographic estimate of the RG flow of an effective QCD coupling see Ref. [34].)

To put the above arguments in favor of an unusually large anomalous dimension for the tetraquark interpolator into perspective, we recall that in AdS/QCD applications to ordinary hadrons anomalous dimensions are generally neglected. This provides a decent approximation as long as the anomalous dimensions of the corresponding two- and three-quark interpolators are much smaller than their classical scaling dimensions. In the UV, the latter is borne out by perturbative QCD results which show a weak, logarithmic RG scaling behavior. In the IR, on the other hand, where much less QCD information is currently available, it seems that anomalous dimensions can become relevant in some channels and generate notable holographic effects, as studied in Ref. [23]. Nevertheless, as a rule one does not expect them to reach magnitudes comparable to the classical scaling dimensions. (Otherwise results of standard AdS/QCD would have to be revised). Our above arguments suggest that the exotic tetraquark channel provides an exception to this rule in which extraordinarily strong dynamical effects (related to good-diquark binding) render the anomalous dimension non-negligible even to leading approximation.

\section{RESULTS AND DISCUSSION}

Several useful and rather generic features of the tetraquark mass spectrum in potentials of the type (20), (21) can be understood without actually solving the eigenvalue problem (10). First, any $\gamma(z)$ which renders the correction (21) negative and decreases the power of $z$ below -2 for $z \rightarrow 0$ would cause a physically unacceptable "collapse to the center" instability of the Sturm-Liouville solutions [32]. In our context, the "center" at $z=0$ corresponds to the $\mathrm{AdS}_{5}$ boundary, and this collapse is avoided by keeping $\eta>0$. An instability may appear even if the singularity remains $\propto 1 / z^{2}$, however. Indeed, as long as the coefficient of this singularity is negative and exceeds a critical magnitude, the negative potential energy

will overcome the stabilizing kinetic (or localization) energy. A straightforward analysis of the small- $z$ behavior of the solutions of Eq. (10) in the potential (20) for general boundary 
dimension $d$ shows that such a collapse is prevented as long as

$$
\frac{d^{2}}{4}+\tilde{\Delta}(\tilde{\Delta}-d)>0
$$

where we abbreviated $\tilde{\Delta} \equiv \Delta+\gamma$. In fact, taking $\tilde{\Delta}$ constant turns the inequality (22) into the Breitenlohner-Freedman (BF) bound $m_{5}^{2} R^{2}>-d^{2} / 4$ [35]. A violation of this bound thus causes the ground-state wavefunction to disintegrate and its eigenvalue to become $m_{0}^{2}=-\infty$. This is a holographic signal for a vacuum instability in the boundary gauge theory. Our form (21) of $\Delta V$, however, which arises from the anomalous-dimension correction (18), has the lower bound

$$
\Delta V(z) \geq-\frac{16}{z^{2}}
$$

(for $d=4$ and all $z$ ). Hence it preserves the BF bound and prevents a collapse from the outset. The inequality (23) is saturated for (constant) $\tilde{\Delta}=2$ or $\gamma=-4$. This generates the lower bound

$$
m_{\bar{q}^{2} q^{2}, 0} \geq m_{\Delta=2,0}=2 \lambda
$$

for the ground-state mass. Therefore $2 \lambda$ is the minimal tetraquark mass which can arise from the bulk mass term (18) with any anomalous dimension. (A coupling of the tetraquark's dual mode to an additional bulk field may release the bound (23) for $z>0$ and thus generate lower masses.)

As a result, we have shown that a suitable ansatz for the tetraquark interpolator's anomalous dimension can indeed generate an extraordinarily large holographic binding energy which drives the lightest tetraquark mass from about $40 \%$ above down to around $20 \%$ below the mass $m_{q \bar{q}, 0}=\sqrt{6} \lambda$ (cf. Eq. (15j) ) of the $\bar{q} q$ ground state. To provide a concrete example for a $\gamma(z)$ of the form (19) which results in a bulk-mode potential with nearly maximal attraction for the ground state, we choose the coefficients inside the narrow range established by the criteria given above as $a=4, b=0.05, \eta=0.001$ and $\kappa=2$. The resulting potential (20) with a typical soft-wall IR scale $\lambda=0.425 \mathrm{GeV}$ is plotted in Fig. 1, (For comparison, we also show the potentials corresponding to the $\Delta=3$ and $\Delta=6$ interpolators without anomalous dimensions, as well as to the one with $\Delta=2$ which saturates the BF bound.) Numerical integration of Eq. (10) in the plotted potential indeed yields a ground-state mass close to the bound (24): $m_{\bar{q}^{2} q^{2}, 0}=2.11 \lambda$. Although the particular ansatz (19) for $\gamma(z)$ is not unique, Fig. 1 reveals that the bounds deduced above determine the behavior of the (almost) maximally attractive potential in a practically model-independent manner. 
We now turn to the radial excitation spectrum of the tetraquark. The masses of the first five excited states (in the potential of Fig. 11) are shown in Fig. 2, In this part of the spectrum, the square masses increase considerably faster than their asymptotic, linear trajectory

$$
m_{n}^{2} \stackrel{n \rightarrow \infty}{\longrightarrow} 4 n \sqrt{\lambda^{4}+b^{2}} \stackrel{|b| \ll \lambda^{2}}{\simeq} 4 \lambda^{2} n
$$

with almost universal slope $4 \lambda^{2}$. In particular, they start to surpass the corresponding masses on the $\bar{q} q$ trajectory around the second excitation level (and those on the uncorrected $\Delta=6$ trajectory from about the fifth excitation onward). As intended, the higherlying tetraquarks can therefore become broad enough to escape detection. Whereas a fully quantitative comparison of Fig. 2 with the experimental scalar mass spectrum as well as a detailed analysis of the numerically generated eigenmodes and decay constants would seem premature in our explorative framework, a few additional and probably rather robust features of the resulting spectrum are noteworthy. Interpreting the second scalar nonet around $1.5 \mathrm{GeV}$ as predominantly the lightest $\bar{q} q$ state would imply the phenomenological mass ratio $m_{\bar{q}^{2} q^{2}, 0} / m_{\bar{q} q, 0} \sim 0.8 / 1.5 \simeq 0.53$ which is somewhat larger than what the tetraquark interpolator's anomalous dimension by itself can generate. (The alternative interpretation of the second nonet as a tetraquark recurrence [7], on the other hand, would raise the question of how to assign the lightest $\bar{q} q$ state.) The remaining part of the ground-state mass ratio may e.g. be supplied by the anomalous dimension of the $\bar{q} q$ interpolator or by couplings of the dual tetraquark modes to additional background fields. An adequate description of molecular-binding or meson-cloud effects could require to take bulk-mode interactions into account, moreover, and the resonance widths will further modify the masses.

A reassuring feature of our anomalous-dimension-mediated tetraquark binding mechanism is that it will work in essentially the same fashion in any of the current AdS/QCD gravity duals. Indeed, $\gamma$ enters the bulk dynamics and hence the tetraquark mode potential only through the mass term of Eq. (11) which universally appears in all AdS/QCD duals [40] and which is model-independently fixed by the AdS/CFT dictionary in Eq. (18). As a consequence, the anomalous-dimension-induced correction (21) and its impact on the tetraquark spectrum are independent of the specific AdS/QCD dynamics under consideration. The $\gamma(z)$ determined above should therefore generate a light scalar tetraquark with substantially more massive radial excitations in any AdS/QCD dual. Nevertheless, it would still be interesting to explore the quantitative impact of the correction (21) in other and 
especially backreacted versions of AdS/QCD (such as Ref. [37]).

The holographic picture of light tetraquarks emerging from our above results can be extended and tested further, e.g. by implementing flavor-symmetry breaking and bulk-mode interactions, analyzing the ensuing decay channels and studying pertinent correlators (which may contain both $\bar{q} q$ and $\bar{q}^{2} q^{2}$ interpolators). It would also be interesting to investigate the behavior of exotic multiquarks in a topological expansion with large numbers of both colors $\left(N_{c}\right)$ and flavors $\left(N_{f}\right)$ where $N_{f} / N_{c}$ is kept fixed [36].

\section{SUMMARY AND CONCLUSIONS}

We have discussed several foundational aspects of light scalar tetraquark physics from a holographic perspective. In particular, we have identified an AdS/QCD representation for the exceptionally large tetraquark binding energy in terms of resolution-dependent bulk-mass corrections for the corresponding dual modes. These corrections are argued to originate (at least in part) from the running anomalous dimension of the four-quark interpolator which encodes the diquark content of the tetraquark. As a consequence, they are independent of the specific AdS/QCD dynamics under consideration. The minimal achievable tetraquark mass is determined by the Breitenlohner-Freedman bound, furthermore, which prevents the tetraquark's dual mode from collapsing into the anti-de Sitter boundary.

In order to provide a quantitative example for this binding mechanism, we have implemented it into the dilaton-soft-wall gravity dual for holographic QCD. The bulk mass corrections which generate nearly maximal attraction in the tetraquark channel are found to be largely determined by consistency and stability requirements, and the minimal tetraquark mass is fixed at twice the dilaton-induced infrared scale. As a result, the generated attraction is indeed strong enough to render the tetraquark the lightest scalar meson, about $20 \%$ lighter than the $\bar{q} q$ ground state. To meet quantitative phenomenological expectations would probably require some additional contribution, however, which may originate e.g. from the anomalous dimension of the $\bar{q} q$ interpolator or from additional bulk fields and interactions. The higher tetraquark excitations turn out to be heavier than their $\bar{q} q$ counterparts, finally, so that their increasing widths (due to increasingly many open decay channels, a larger phase space and potential dynamical enhancements) should prevent them from generating more low-lying scalar resonances than experimentally seen. 
We would like to thank Dietmar Ebert and Eberhard Klempt for their questions regarding a holographic description of light scalar tetraquarks. Financial support from the Deutsche Forschungsgemeinschaft (DFG) is also acknowledged.

[1] F.E. Close and N.A. Törnqvist, J. Phys. G 28, R249 (2002).

[2] C. Amsler and N.A. Törnqvist, Phys. Rep. 389, 61 (2004).

[3] E. Klempt and A. Zaitsev, Phys. Rep. 454, 1 (2007).

[4] C. Amsler et al., Review of Particle Physics, Phys. Lett. B 667 (2008) 1.

[5] C. McNeile, Nucl. Phys. B (Proc. Suppl.) 186, 264 (2009); T. Kunihiro et al., Nucl. Phys. B (Proc. Suppl.) 186, 294 (2009); S. Prelovsek et al., arXiv:1002.0193 [hep-ph].

[6] R.L. Jaffe, Phys. Rev. D 15, 267, 281 (1977).

[7] L. Maiani, F. Piccinini, A.D. Polosa and V. Riquer, Eur. Phys. J. C 50, 609 (2007).

[8] L. Maiani, F. Piccinini, A.D. Polosa and V. Riquer, Phys. Rev. Lett. 93, 212002 (2004).

[9] T.V. Brito, F.S. Navarra, M. Nielsen and M.E. Bracco, Phys. Lett. B 608, 69 (2005); H.-J. Lee and N.I. Kochelev, Phys. Lett. B 642, 358 (2006); H. X. Chen, A. Hosaka, and S. L. Zhu, Phys. Lett. B 650, 369 (2007); H.-J. Lee and N.I. Kochelev, Phys. Rev. D 78, 076005 (2008); D. Ebert, R.N. Faustov and V.O. Galkin, Eur. Phys. J. C 60, 273 (2009).

[10] R.L. Jaffe, Phys. Rep. 409, 1 (2005); arXiv:hep-ph/0701038.

[11] G 't Hooft, G. Isidori, L. Maiani, A.D. Polosa and V. Riquer, Phys. Lett. B 662, 424 (2008).

[12] O. Aharony et al., Phys. Rep. 323, 183 (2000);

[13] K. Peeters and M. Zamaklar, Eur. Phys. J. Special Topics 152, 113 (2007); S.J. Brodsky and G.F. de Téramond, arXiv:0802.0514; J. Erdmenger, N. Evans, I. Kirsch and E. Threlfall, Eur. Phys. J. A 35, 81 (2008).

[14] A. Vega and I. Schmidt, Phys. Rev. D 78, 017703 (2008).

[15] P. Colangelo, F. De Fazio, F. Giannuzzi, F. Jugeau and S. Nicotri, Phys. Rev. D 78, 055009 (2008).

[16] W. de Paula and T. Frederico, arXiv:0908.4282 [hep-ph].

[17] H. Boschi-Filho and N.R.F. Braga, J. High Energy Phys. 05 (2003) 009; Eur. Phys. J. C 32, 529 (2004); G.F. de Téramond and S.J. Brodsky, Phys. Rev. Lett. 94, 201601 (2005); H. Boschi-Filho, N.R.F. Braga, and H.L. Carrion, Phys. Rev. D 73, 047901 (2006); P. Colangelo, 
F. De Fazio, F. Jugeau, and S. Nicotri, Phys. Lett. B 652, 73 (2007); H. Forkel, Phys. Rev. D 78, 025001 (2008); Proc. Sci., CONFINEMENT 8, 184 (2008), arXiv:0812.3881]; arXiv:0808.0304 [hep-ph].

[18] U.G. Meißner, Comments Nucl. Part. Phys. 20, 119 (1991).

[19] T. Schäfer, E.V. Shuryak and J.J.M. Verbaarschot, Nucl. Phys. B 412, 143 (1994).

[20] T. Schäfer, Phys. Rev. D 68, 114017 (2003).

[21] M. Cristoforetti, P. Faccioli, E.V. Shuryak and M. Traini, Phys. Rev. D 70, 054016 (2004).

[22] M. Shifman and A. Vainshtein, Phys. Rev. D 71, 074010 (2005); A. Selem and F. Wilczek, MIT-CTP-3721, arXiv:hep-ph/0602128.

[23] H. Forkel and E. Klempt, Phys. Lett. B 679, 77 (2009).

[24] A. Karch, E. Katz, D.T. Son and M.A. Stephanov, Phys. Rev. D 74, 015005 (2006).

[25] J. Erlich, E. Katz, D.T. Son and M.A. Stephanov, Phys. Rev. Lett. 95 (2005) 261602 arXiv:hep-ph/0501128; L. Da Rold and A. Pomarol, Nucl. Phys. B 721 (2005) 79 arXiv:hep-ph/0501218.

[26] A. Manohar, arXiv:hep-ph/9607484.

[27] J.R. Peláez, Phys. Rev. Lett. 92, 102001 (2004); M. Uehara, arXiv:hep-ph/0401037; J. Nebreda and J.R. Peláez, arXiv:1002.1271; and references therein.

[28] E. Santopinto and G. Galata, Phys. Rev. C 75, 045206 (2007).

[29] M. Abramowitz and I.A. Stegun, Handbook of Mathematical Functions (U.S. GPO, Washington, DC, 1972).

[30] H. Forkel, M. Beyer and T. Frederico, JHEP 07, 077 (2007) arXiv:0705.1857]; Intl. J. Mod. Phys. E 16, 2794 (2007) arXiv:0705.4115]; H. Forkel, Proc. Sci., QCD-TNT09 (2009) 014 arXiv:0910.5955].

[31] D.V. Bugg, Phys. Rept. 397, 257 (2004) hep-ex/0412045.

[32] L.D. Landau and E.M. Lifshitz, Quantum mechanics, non-relativistic theory (3rd edition, Pergamon Press, Oxford 1991).

[33] W. Mück, arXiv:1006.2987.

[34] S.J. Brodsky, G.F. de Téramond and A. Deur, Phys. Rev. D 81, 096010 (2010); arXiv:1002:4660.

[35] P. Breitenlohner and D.Z. Freedman, Ann. Phys. 144, 249 (1982).

[36] G. Veneziano, Nucl. Phys. B 117, 519 (1976). 
[37] W. de Paula, T. Frederico, H. Forkel and M. Beyer, Phys. Rev. D 79, 075019 (2009) arXiv:0806.3830 [hep-ph]]; Proc. Sci., LC2008, 046 (2008) [arXiv:0810.2710 [hep-ph]].

[38] One should keep in mind, however, that alternative descriptions in terms of molecular resonances (see e.g. Ref. [18] and the cited reviews) are difficult to distinguish both in QCD [10] and holographically.

[39] We shall tentatively assume that the RG mixing among anomalous dimensions remains small in the restricted $z$ (resp. $\mu$ ) range $z \lesssim 1 / \lambda$ of interest.

[40] This is because the geometry of AdS/QCD duals approaches exact $\operatorname{AdS}_{5}$ for $z \rightarrow 0$ in order to ensure conformality of the dual gauge theory in the UV. 


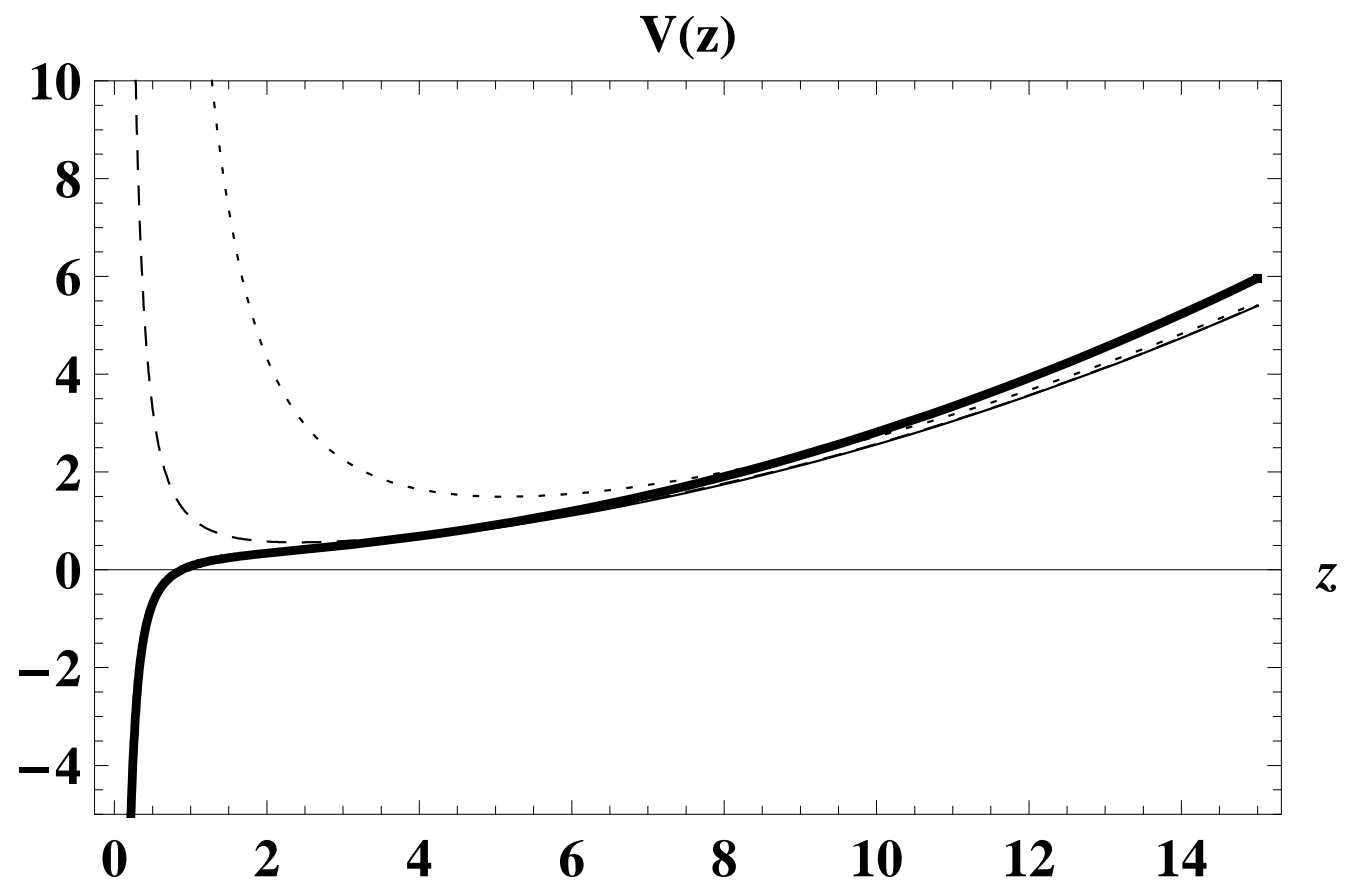

FIG. 1: The Sturm-Liouville potential (20) of the tetraquark's dual mode for the anomalous dimension (19) with $a=4, b=0.05, \eta=0.001$ and $\kappa=2$ (thick line). For comparison, we also plot the potential for the four-quark interpolator with $\Delta=6, \gamma=0$ (dotted line) and for the $\bar{q} q$ interpolator with $\Delta=3$ (dashed line). In addition, we plot the potential for $\Delta=2$ (thin line) which saturates the Breitenlohner-Freedman bound. 


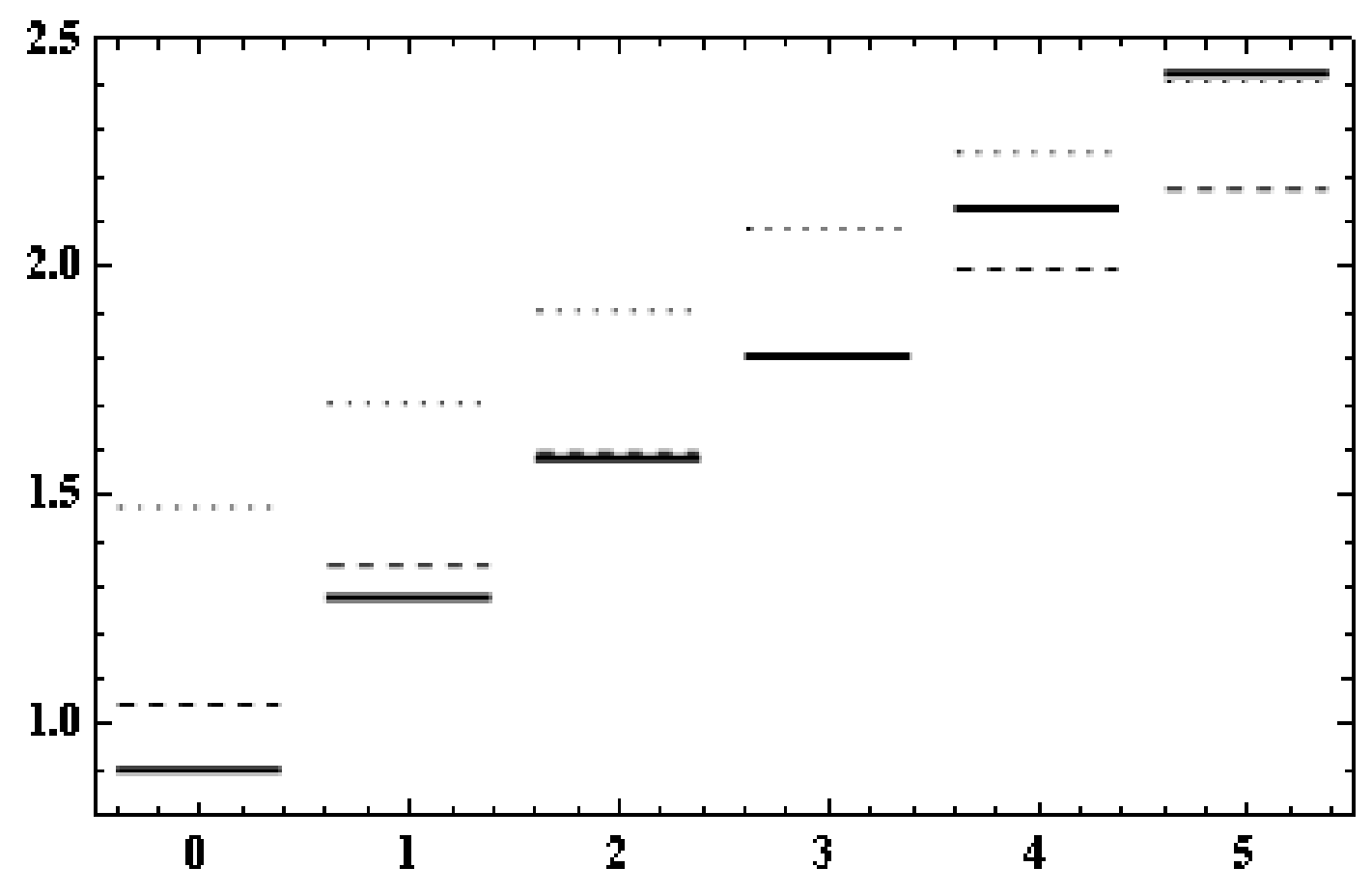

FIG. 2: The ground state $(n=0)$ and the first five excitations of the tetraquark mass spectrum in the potential of Fig. 1 (thick bars). We further plot the spectra for the $\bar{q} q$ interpolator (Eq. (15), dashed bars) and for the $\Delta=6$ interpolator (Eq. (16), dotted bars) with vanishing anomalous dimensions. 